\title{
Enfermedad inflamatoria intestinal en pacientes celíacos
}

\author{
M. Masachs, F. Casellas y J. R. Malagelada \\ Unitat d'Atenció Crohn-Colitis. Hospital Universitari Vall d'Hebron. Barcelona
}

\section{RESUMEN}

Introducción: se ha sugerido una potencial asociación entre la enfermedad celíaca y la enfermedad inflamatoria intestinal, que puede justificar que ambas enfermedades puedan presentarse en un mismo enfermo o en sus familiares de primer orden con mayor frecuencia de lo esperado.

Objetivo: determinar la prevalencia de la enfermedad de Crohn y la colitis ulcerosa en los enfermos celíacos y en sus familiares.

Método: estudio epidemiológico prospectivo transversal en un grupo de pacientes celíacos, sus familiares de primer grado y un grupo control de características epidemiológicas similares, constituido por familiares de pacientes que acuden al Servicio de Urgencias por un problema agudo. Para detectar la existencia de colitis ulcerosa y enfermedad de Crohn en los celíacos y sus familiares, se realizó una entrevista semiestructurada.

Resultados: se han incluido 86 celíacos y 432 familiares, que se han comparado con 809 controles (129 pacientes con una enfermedad aguda y 680 familiares de primer grado suyos). Se han detectado 3 casos de enfermedad de Crohn en el grupo de los enfermos celíacos y 4 casos de enfermedad de Crohn en sus familiares. Sólo se ha detectado 1 caso de enfermedad de Crohn en el grupo control $(p<0,01)$. No se ha identificado ningún caso de colitis ulcerosa en ninguno de los tres grupos de estudio.

Conclusión: los pacientes con enfermedad celíaca y sus familiares tienen mayor predisposición a presentar una enfermedad de Crohn, que la población control.

Palabras clave: Enfermedad celíaca. Enfermedad de Crohn. Colitis ulcerosa. Epidemiología. Prevalencia.

\begin{abstract}
Introduction: a potential association between celic disease and inflammatory bowel disease hs been suggested, which may explain the fact that both disorders occasionally present in one patient or in his/her first-degree relatives more frequently than expected.
\end{abstract}

Objective: to establish the prevalence of Crohn's disease and ulcerative colitis in celiac patients and their relatives.

Method: a cross-sectional, prospective epidemiological study in a group of celiac patients, their first-degree relatives, and a control group with similar epidemiological characteristics including the relatives of patients presenting at the ER for acute conditions. A semistructured interview was used to identify the presence of Crohn's disease and ulcerative colitis in celiac patients and their relatives.

Results: in all, 86 celiac patients and 432 relatives were included, who were compared to 809 control subjects (129 patients with acute conditions and 680 first-degree relatives). Three cases of Crohn's disease were identified among celiac patients, and 4 cases among their relatives. Only 1 case of Crohn's disease was detected in the control group $(p<0.01)$. No cases of ulcerative colitis were detected in any of the study groups.

Conclusion: patients with celiac disease and their relatives have a greater predisposition to Crohn's disease versus the control population.

Key words: Celiac disease. Crohn's disease. Ulcerative colitis. Epidemiology. Prevalence.

Masachs M, Casellas F, Malagelada JR. Enfermedad inflamatoria intestinal en pacientes celíacos. Rev Esp Enferm Dig 2007; 99: 446-450.

Financiado en parte por 2005SGR00398 de la Generalitat de Catalunya, Departament d'Universitats, Recerca i Societat de l'Informació.

Recibido: 26-02-07.

Aceptado: 12-03-07.

Correspondencia: Frances Casellas. Servei de Digestiu. Hospital Universitari Vall d'Hebron. Passeig Vall d'Hebron, 119. 08035 Barcelona. e-mail: fcasellas@vhebron.net

\section{INTRODUCCIÓN}

La enfermedad celíaca se define como la enteropatía autoinmune inducida por la ingesta de gluten en individuos genéticamente predispuestos, caracterizada por la existencia de lesiones intestinales sugestivas que regresan con la exclusión del gluten de la dieta $(1,2)$. Las lesiones histológicas, son el resultado de una combinación 
de factores ambientales (gluten) y genéticos (3) en relación con los genes HLA de clase II DQ2 y DQ8 del brazo corto del cromosoma 6 que activan los linfocitos TH1 intestinales específicos. En nuestro medio el HLA-DQ2 se expresa en el $88-94 \%$ de los pacientes, expresando el HLA-DQ8 el resto (4). La relación entre la expresión de ciertos alelos de los heterodímeros HLA-DQ2 y HLADQ8 y la enfermedad celíaca justifica la existencia de una predisposición familiar, de forma que alrededor del $5 \%$ de los familiares de primer grado $(4,5)$ e incluso de segundo grado (6) desarrollan la enfermedad.

Una de las características de la enfermedad celíaca es su asociación con el desarrollo de anticuerpos. Algunos de ellos, como los antigliadina, los antiendomisio y los antitransglutaminasa tisular, tienen un gran valor diagnóstico y han causado un cambio profundo tanto en la epidemiología como en el reconocimiento de las manifestaciones clínicas de la enfermedad. Gracias a su diagnóstico serológico, la celiaquía ha pasado de ser una enfermedad rara de niños con malnutrición a ser una enfermedad frecuente, que se presenta a cualquier edad, tanto niños como adultos y ancianos (7), puede manifestarse con síntomas digestivos o extradigestivos y diagnosticarse incluso en fase asintomática. En nuestro medio la prevalencia actual de la celiaquía oscila entre 1/118 en la población infantil y 1/389 en la población adulta (8-10).

Por su parte, la enfermedad inflamatoria intestinal (EII) es una inflamación crónica de causa no específica del intestino grueso y/o el resto del tracto digestivo, de etiología desconocida, curso recidivante y que se asocia a complicaciones locales y/o sistémicas. El término EII engloba dos entidades, la colitis ulcerosa y la enfermedad de Crohn. Al igual que en la enfermedad celíaca, en la etiopatogenia de la EII interaccionan factores inmunológicos, genéticos y ambientales (11-13). Presenta una prevalencia alta en Europa, habiendo demostrado un estudio multicéntrico Europeo una incidencia de 10,4 y 5,6 casos $/ 10^{5}$ hab./año, para la colitis ulcerosa y la enfermedad de Crohn, respectivamente (14).

Ambas entidades, la enfermedad celíaca y la EII, comparten múltiples analogías. Las dos se asocian a enfermedades de base genética e inmunoinflamatoria, comparten una respuesta inmune similar relacionada con un patrón de respuesta linfocitaria Th1 (15), cursan con una inflamación crónica del intestino, y su patogenia es el resultado de la interacción entre factores inmunológicos, genéticos y ambientales. Se ha sugerido que la enfermedad celíaca y la enfermedad inflamatoria intestinal puedan tener alguna relación y presentarse conjuntamente en un mismo enfermo o más predominantemente en los familiares. En este sentido, se han realizado dos estudios caso control, uno en Italia y otro en Estados Unidos que muestran que la EII es más común en los celíacos que en la población general $(16,17)$. Además, varios casos aislados publicados o series de casos han sugerido una asociación entre la enfermedad celíaca y la EII, más frecuentemente colitis ulcerosa (CU) $(18,19)$.
Basados en la hipótesis que la prevalencia de la EII es más elevada en los enfermos celíacos y sus familiares, se ha diseñado un estudio prospectivo transversal con el objetivo de determinar la prevalencia de la EII, tanto de colitis ulcerosa como de enfermedad de Crohn, en los celíacos y sus familiares, para compararla con la de un grupo poblacional control y sus familiares de primer grado.

\section{MATERIAL Y MÉTODO}

\section{Pacientes}

Se han incluido en el estudio tres grupos de participantes: pacientes celíacos, familiares de primer grado de los pacientes y un grupo control. El grupo de celíacos estaba formado por todos los enfermos celíacos, diagnosticados previamente según los criterios vigentes, mediante serología y biopsia yeyunal compatible (30), que acudieron durante el período de un año a la Unidad de Pruebas Funcionales Digestivas del Hospital Universitari Vall d'Hebron. El grupo de familiares celíacos lo formaban los familiares de primer grado de los enfermos celíacos. El grupo control estaba formado por pacientes con características epidemiológicas similares a los del grupo de enfermos celíacos, que acudieron al servicio de urgencias por un problema agudo de salud (como cefalea, infección respiratoria, cólico nefrítico, urticaria,...) y un subgrupo formado por sus familiares de primer grado.

\section{Procedimiento}

Durante el periodo de un año, todos los pacientes que eran atendidos en el servicio de nuestro hospital con una biopsia diagnóstica de enfermedad de celíaca eran preguntados para participar en el estudio para determinar si presentaban enfermedad inflamatoria intestinal y si era presente en sus familiares de primer grado. Los pacientes celíacos y sus familiares eran entrevistados mediante un cuestionario semiestructurado, en el mismo hospital o por teléfono. Los controles eran entrevistados mediante un cuestionario semiestrucurado que se daba mientras estaban en el servicio de urgencias. Se recogieron datos clínicos, epidemiológicos y la existencia de enfermedad de Crohn o colitis ulcerosa de acuerdo con el conocimiento de que se supiera diagnosticado, seguía tratamiento para la enfermedad inflamatoria intestinal y controlado por digestólogo por dicho motivo.

\section{Estadística}

Las variables socio-demográficas y datos clínicos se han expresado mediante medianas y percentiles 25 y 75 . La prevalencia para la colitis ulcerosa y la enfermedad de Crohn se determinó de forma independiente para cada 
uno de los tres grupos de participantes. Las diferencias entre medianas se determinaron según las pruebas de Mann-whitney o de Kruskal-Wallis. La existencia de diferencias de variables cualitativas se determinó mediante el test exacto de Fisher. El nivel de significación estadística aceptado fue el menor del 0,05.

\section{RESULTADOS}

\section{Participantes en el estudio}

En el estudio han participado un total de 1.357 sujetos (116 celíacos, 432 familiares y 809 controles). De los 116 pacientes que cumplían criterios para su inclusión en el grupo de los enfermos celíacos, se han excluido 30 por no poderse realizar la entrevista. El grupo familiares de primer grado de celíacos estaba integrado por 432 familiares, con un promedio de 5 familiares incluidos por paciente. El grupo control estaba constituido por 809 personas, 129 pacientes con una enfermedad aguda y 680 familiares de primer grado suyos, también con un promedio de 5 familiares incluidos por paciente control. Las características de los participantes incluidos en el estudio se indican en la tabla I. Todos los pacientes celíacos seguían dieta sin gluten, estando en el momento de la inclusión libres de síntomas y sin diarrea ( 2 deposiciones diarias). El seguimiento de la dieta era correcto en 80 pacientes, refiriendo los 6 restantes un consumo intencional o no intencional de gluten de forma ocasional. Tres pacientes seguían tratamiento con corticoides u otros inmunosupresores por presentar enfermedad celíaca refractaria.

\section{Prevalencia de la enfermedad inflamatoria intestinal}

Como muestra la figura 1 se han detectado 3 casos de enfermedad de Crohn en el grupo de enfermos celíacos, 4 casos de enfermedad de Crohn en el grupo de los familiares de celíacos y 1 caso aislado de enfermedad de Crohn en el grupo de 809 controles (Fig. 1). El riesgo relativo para enfermedad de Crohn en los pacientes celíacos es de 27,3, con un intervalo de confianza del $95 \%$ entre 2,8-259,5. El riesgo relativo para enfermedad de Crohn en los familiares de pacientes celíacos es de 7,5, con un intervalos de confianza del $95 \%$ entre 0,8-66,8. Los 4 casos de enfermedad de Crohn detectados en el grupo de familiares de celíacos corresponden en cada caso a un hermano del paciente celíaco. No se ha identificado ningún caso de colitis ulcerosa en ninguno de los tres grupos de estudio. Los tres enfermos celíacos y con enfermedad de Crohn detectados corresponden al fenotipo A1L1B2 (edad del diagnóstico por debajo de los 40 años, localizado en íleon terminal y con patrón estenosante) fenotipo de la clasificación de Montreal de la enfermedad de Crohn (31).

La prevalencia de enfermedad de Crohn en los pacientes celíacos (3.488 por 100.000 habitantes) es significati-
Tabla I. Principales características de los pacientes con enfermedad celíaca incluidos en el estudios, expresadas en valor absoluto o mediana (percentil 25 - percentil 75)

\begin{tabular}{|c|c|}
\hline & Grupo enfermos celíacos \\
\hline Número & 86 \\
\hline Edad & 34 [29-55] \\
\hline $\operatorname{Sexo}(M / V)$ & $59 / 27$ \\
\hline Tiempo evolución (años) & $6[2-21]$ \\
\hline Anemia ferropénica (S/N) & $20 / 59$ \\
\hline Tratamiento & $\begin{array}{c}\text { Dieta sin gluten: } 86 \\
\text { Tratamiento esteroideo: } 1 \\
\text { Tratamiento inmunosupresor: } 2\end{array}$ \\
\hline Enfermedades asociadas & $\begin{array}{c}\text { Hipoplasia dental: } 6 \\
\text { Diabetes tipo I: } 1 \\
\text { Déficit de IgA: } 3 \\
\text { Dermatitis herpetiforme: } 8 \\
\text { Tiroiditis: } 7 \\
\text { Hipertransaminasemia: } 2 \\
\text { Epilepsia: } 1 \\
\text { Polineuropatía: } 1\end{array}$ \\
\hline Seguimiento de la dieta & $\begin{array}{l}\text { Correctamente seguida: } 80 \\
\text { Incumplimiento no intencional: } 2 \\
\text { Incumplimiento intencional: } 4\end{array}$ \\
\hline
\end{tabular}

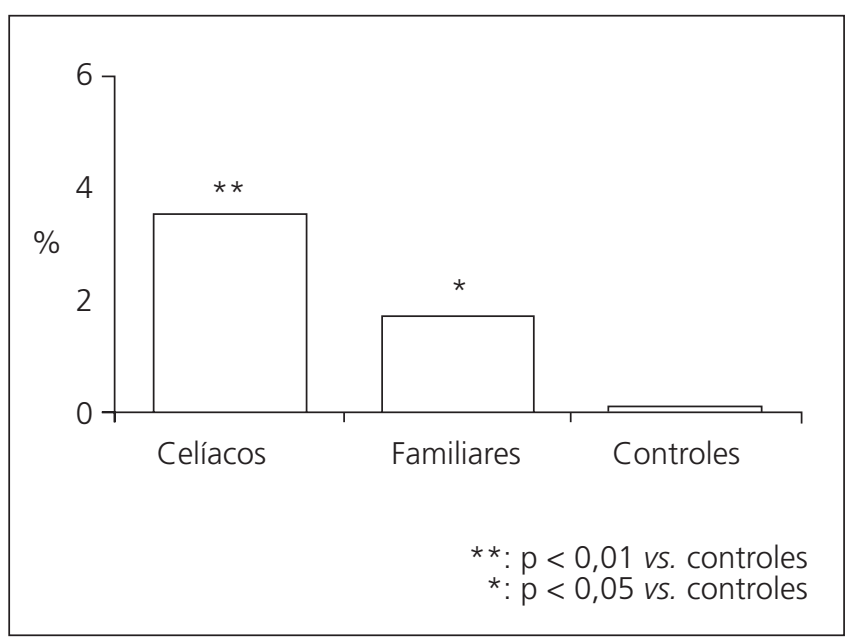

Fig. 1. Porcentaje de casos de enfermedad de Crohn reconocidos en los tres grupos de participantes (pacientes celíacos, familiares de primer grado de los pacientes y controles). La tasa de casos de enfermedad de Crohn en los grupos pacientes celíacos y familiares fue significativamente superior a la del grupo control.

vamente más alta que el grupo control (124 por 100.000 habitantes, $\mathrm{p}<0,01)$. De forma similar, la prevalencia de la enfermedad de Crohn en los familiares de primer grado de los pacientes celíacos (926 por 100.000 habitantes) es también estadísticamente significativa más alta que el grupo control $(\mathrm{p}<0,05)$. En contraste, la prevalencia de colitis ulcerosa en pacientes celíacos y sus familiares de primer grado, no eran diferentes que las obtenidas en el grupo control. 


\section{DISCUSIÓN}

Basado en la hipótesis que la enfermedad inflamatoria intestinal es más frecuente en los enfermos celíacos y sus familiares, se ha planteado un estudio con el objetivo de determinar la prevalencia de la enfermedad de Crohn y la colitis ulcerosa en los celíacos y sus familiares, y compararlo con los obtenidos de una muestra de la población control. Los resultados del presente estudio observacional, transversal y prospectivo con 86 pacientes celíacos, 432 familiares y 809 controles han demostrado una prevalencia de enfermedad de Crohn en los celíacos y sus familiares significativamente superior a la esperada. No ha sucedido lo mismo con la colitis ulcerosa, que no ha demostrado una prevalencia estadísticamente diferente a la esperada en los enfermos celíacos ni en sus familiares.

En nuestro estudio la prevalencia de la enfermedad de Crohn en los controles ha sido de 124 casos por 100.000 habitantes, valor muy parecido al descrito en la población española de 116 casos por 100.000 habitantes (32), lo que da fiabilidad al grupo control escogido. La prevalencia de enfermedad de Crohn observada en nuestro estudio en los grupos de pacientes celíacos y de sus familiares es de 3.488 por 100.000 y de 926 por 100.000 respectivamente. Aunque no se puedan hacer comparaciones estadísticas con los valores descritos para la población española publicados previamente, la prevalencia de la enfermedad de Crohn en los pacientes con enfermedad celíaca y en sus familiares es muy elevada, cifrándose en al menos 8 veces los valores publicados para la población española.

La predisposición de los celíacos y sus familiares a desarrollar enfermedad de Crohn puede tener importantes repercusiones clínicas, como el hecho de tener presente siempre la posibilidad diagnóstica de enfermedad de Crohn en aquellos pacientes con enfermedad celíaca que no se controlan adecuadamente con la dieta sin gluten o presentan productos patológicos en heces. Del mismo modo, en los familiares de pacientes con enfermedad celíaca, debería también considerarse la posibilidad de una enfermedad de Crohn ante la aparición de síntomas digestivos compatibles.

El mecanismo de la potencial asociación entre enfermedad celíaca y enfermedad Crohn no puede inferirse del presente estudio. Ambas enfermedades se caracterizan por la pérdida de tolerancia a antígenos de la luz intestinal (al gluten o a la microbiota intestinal respectivamente). En el desarrollo de la lesión intestinal en ambas enfermedades están implicados la sobreexpresión local de interleucina-15 y la activación de linfocitos T CD4+ con un patrón Th1 de citocinas (33). Este patrón de respuesta inmune no es tan característico de la colitis ulcerosa, más relacionada con un patrón Th2, lo que quizás podría explicar la ausencia de relación observada en nuestro estudio entre enfermedad celíaca y colitis ulcerosa.

En conclusión, los resultados del presente estudio permiten sugerir la existencia de una asociación entre la enfermedad celíaca y la enfermedad de Crohn, con una pre- valencia más alta de lo esperada tanto en los pacientes con enfermedad celíaca como en sus familiares de primer grado.

\section{BIBLIOGRAFÍA}

1. Ciclitira PJ. AGA technical review on Celiac Sprue. Gastroenterol 2001; 120: 1526-40.

2. American Gastroenterological Association medical position statement: Celiac sprue. Gastroenterol 2001; 120: 1522-5.

3. Stern M, Ciclitira PJ, van Eckert R, Feighery C, Janssen FW, Méndez $\mathrm{E}$, et al. Analysis and clinical effects of gluten in coeliac disease. Eur J Gastroenteol Hepatol 2001; 13: 741-7.

4. Farré C, Humbert P, Vilar P. Catalonian Coeliac Disease Study Group. Serological markers and HLA-DQ2 haplotype among first-degree relatives of celiac patients. Dig Dis Sci 199; 44: 2344-9.

5. López Hoyos M, Bartolomé MJ, Castro B, et al. Cribado de la enfermedad celíaca en familiares de primer grado. Med Clin 2003; 120: 132-4.

6. Rodrigo L, Riestra S, Fuentes D, González S, López Vázquez A, López Larrea C. Diverse clinical presentation of celiac disease in the same family. Rev Esp Enferm Dig 2004; 96: 416-9.

7. Fasano A, Catassi C. Current approaches to diagnosis and treatment of celiac disease: An evolving spectrum. Gastroenterol 2001; 120: 636-51.

8. Riestra S, Fernandez E, Rodrigo L, García S, Ocio G. Prevalence of coeliac disease in the general population of northern Spain. Strategies of serologic screening. Scand J Gastroenterol 2000; 35: 398-402.

9. Cilleruelo ML, Román E, Jiménez J, et al. Enfermedad celíaca silente: Explorando el iceberg en población escolar. An Esp Pediatr 2002; 57: 321-6.

10. Castaño L, Blarduni E, Ortiz L, Núñez J, Bilbao JR, Rica I, et al. Prospective population screening for celiac disease: High prevalence in the first 3 years of life. J Pediatr Gastroenterol Nutr 2004; 39: 80-4.

11. Farrell RJ, Peppercorn MA. Ulcerative Colitis. Lancet 2002; 359: $331-40$.

12. Shanahan F. Probiotics and inflammatory bowel disease: From fads and fantasy to facts and future. Br J Nutr 2002; 88 (Supl. 1): S5-S9.

13. Fiocchi C. Inflammatory bowel disease: Etiology and pathogenesis. Gastroenterol 1998; 115: 182-205.

14. Shivananda S, Lennard-Jones J, Logan R, Fear N, Price A, Carpenter $\mathrm{L}$, et al. Incidence of inflammatory bowel disease across Europe: Is there a difference between north and south? Results of the European Collaborative study on Inflammatory Bowel Dis (EC-IBD). Gut 1996; 39: 690-7.

15. Radojevic N, McKay DM, Merger M, Vallance BA, Collins SM, Croitoru K. Characterization of enteric functional changes evoked by in vivo anti-CD3 cell activation. Am J Physiol 1999; 276: 715-23.

16. Cottone M, Marrone C, Casà A, Oliva L, Orlando A, Calabrese E, et al. Familial Occurrence of inflammatory Bowel Disease in Celiac Disease. Inflam Bowel Dis 2003; 9: 321-3.

17. Yang A, Chen Y, Scherl E, Neugut A, Bhagat G, Green P. Inflammatory Bowel Disease in Patients with Celiac Disease. Inflam Bowel Dis 2005 ; $11: 528-32$.

18. Sahah A, Mayberry JF, Williams G, Holt P, Loft De Rhodes. Epidemiological survey of coeliac disease and inflammatory bowel disease in first-degree relatives of coeliac patients. Q J Med 1990; 74: 283-8.

19. Bulger K, Griffin M, Dervan P, Lennon J, Crowe J. Coeliac disease in association with inflammatory bowel disease. Postgrad Med J 1988; 64: 336 .

20. Kitis G, Holmes GK, Cooper BT, Thompson H, Allan RN. Association of coeliac disease and inflammatory bowel disease. Gut 1980; 21: 636-41.

21. Gillberg R, Dotevall G, Ahren C . Chronic inflammatory bowel disease in patients with coeliac disease. Scand J Gastroenterol 1982; 17: 491-6.

22. Euler AR, Ament ME. Celiac sprue and Crohn's disease: An association causing severe growth retardation. Gastroenterol 1977; 72: 729-31.

23. Kumar PJ, O'Donoghue DP, Gibson J, Stansfeld A, Dawson AM. The existence of inflammatory bowel lesions in gluten-sensitive enteropathy. Postgrad Med J 1979; 55: 753-6. 
24. Breen EG, Coughlan G, Connolly CE, Stevens FM, McCarthy CF. Coeliac proctitis. Scand J Gastroenterol 1987; 22: 247-477.

25. Breen EG, Coghlan G, Connolly EC, Stevens FM, McCarthy CF. Increased association of ulcerative colitis and celiac disease. Ir J Med Sci 1987; 156: 120-1.

26. Mayberry JF, Smart HL, Toghill PJ. Familial association between celiac disease and ulcerative colitis: Preliminary communication. J R Soc Med 1986; 79: 204-5.

27. Falchuk KR, Falchuk ZM. Selective immunoglobulin a deficiency, ulcerative colitis, and gluten-sensitive enterophaty- a unique association. Gastroenterol 1975; 69: 503-6.

28. Sood A, Midha V, Sood N, Avasthi G, Kaushal V. Coexistence of celiac disease and ulcerative colitis. Indian Gastroenterol 2001; 20: 200-1.

29. Habior A, Rawa T, Orlowska J, Sankowska M, Lewartowska A, Tilszer A, et al. Association of primary slerosing cholangitis, ulcerative colitis and coeliac disease in female siblings. Eur J Gastroenterol Hepatol 2003; 14: 787-91.
30. NIH Consensus Development Conference Statement: Celiac Disease. 2004. Available at: http://consensus.nih.gov/cons/118/118celiacPDF. pdf Accessed 30 September 2004.

31. Silverg M, Satsangi J, Ahmad T, Ian D, Bernstein C, Brant S, et al. Toward an integrated clinical, molecular and serological classification of inflammatory bowe disease: Report of a Working Party of the 2005 Montreal World Congress of Gastroenterology. Can J Gatroenterol 2005; 19 (Supl. A): 5A-36A.

32. Saro C, Lacort M, Arguelles G, Antón Magarzo J, García López R, Navascues CA, et al. Incidence and prevalence of inflammatory bowel disease in Gijon, Asturias, Spain. Gastroenterol Hepatol 2000; 23: $322-7$

33. Di Sabatino A, Ciccocioppo R, Cupelli F, Cinque B, Millimaggi $\mathrm{D}$, Clarkson M, et al. Epithelium-derived interleukin-15 regulates intraepithelial lymphocyte Th1 cytokine production, cytotoxicity and survival in coeliac disease. Gut doi: 10.1136/gut. 2005.068684 . 